\title{
DIRAC COHOMOLOGY AND CHARACTER LIFTING
}

\author{
JING-SONG HUANG
}

\begin{abstract}
The endoscopic transfer factor is expressed as difference of characters for the even and odd parts of the spin modules, or Dirac index of the trivial representation. The lifting of tempered characters in terms of index of Dirac cohomology is calculated explicitly.
\end{abstract}

\section{INTRODUCTION}

The lifting of representations between reductive algebraic groups plays an important role in representation theory. The endoscopic transfer in Langlands functionality and the theta correspondence in Howe's reductive dual pairs are primary examples.

Dirac operators are employed for geometric construction of discrete series by Parthasarathy [30], Atiyah and Schmid [6], and tempered representations by Wolf [42]. In the late 1990's, Vogan made a conjecture on the algebraic property of the Dirac operators in Lie algebra setting. This conjecture was proved by Pandzic and myself in 2002 [18]. This led us to study Dirac cohomology of Harish-Chandra modules [19]. Kostant extended the concept of Dirac cohomology and Vogan's conjecture to the more general setting of the cubic Dirac operator [24].

In the formulation of central problems in Langlands program stable conjugacy plays an pivotal role. The theory of endoscopy investigates the difference between orbital integral over ordinary and stable conjugacy classes. As Dirac cohomology of a Harish-Chandra module determines its K-character [22], it corresponds to the dual object of the orbital integral on elliptic elements. In this note we focus on using Dirac cohomology for endoscopic transfer. The transfer factor is difference of characters for the even and odd parts of the spin modules, or Dirac index of the trivial representation [15]. The aim of this note is to extend the calculation of lifting of characters to tempered representations with nonzero Dirac cohomology. The ultimate goal is to understand lifting of characters for all unitary representations with nonzero Dirac cohomology (the Dirac series).

Jeff Adams [1] defined a lifting on characters between orthogonal groups and nonlinear metaplectic groups over real numbers, and it was extended to the p-adic case by Tatiana Howard [13]. This lifting of characters is closely related to both endoscopy and theta correspondence, which also appears in the work of David Renard [31] and Wen-Wei Li [29]. The Adams lifting

2010 Mathematics Subject Classification. Primary 22E47; Secondary 22E46.

Key words and phrases. Dirac cohomology, Dirac series, cubic Dirac operators, endoscopic transfer, character lifting.

The research described in this paper is supported by grants No. 16303218 from Research Grant Council of HKSAR. 
of characters for orthogonal and symplectic groups is given by the formal difference of the oscillator representations of metaplectic groups and are related to the symplectic Dirac cohomology for Lie superalgebra [20]. Since the symplectic Dirac cohomology of modules for Lie superalgebras is very different from the Dirac cohomology of Harish-Chandra modules, we deal with the transfer factor of the Adams lifting in another paper [16].

\section{Preliminaries on Dirac cohomology}

For a real reductive group $G$ with a Cartan involution $\theta$, denote by $\mathfrak{g}_{0}$ its Lie algebra and assume that $K=G^{\theta}$ is a maximal compact subgroup of $G$. Let $\mathfrak{g}=\mathfrak{k} \oplus \mathfrak{p}$ be the Cartan decomposition for the complexified Lie algebra of $G$. Let $B$ be a non-degenerate invariant symmetric bilinear form on $\mathfrak{g}$, which restricts to the Killing form on the semisimple part $[\mathfrak{g}, \mathfrak{g}]$ of $\mathfrak{g}$.

Let $U(\mathfrak{g})$ be the universal enveloping algebra of $\mathfrak{g}$ and $C(\mathfrak{p})$ the Clifford algebra of $\mathfrak{p}$ with respect to $B$. Then one can consider the following version of the Dirac operator:

$$
D=\sum_{i=1}^{n} Z_{i} \otimes Z_{i} \in U(\mathfrak{g}) \otimes C(\mathfrak{p}) ;
$$

here $Z_{1}, \ldots, Z_{n}$ is an orthonormal basis of $\mathfrak{p}$ with respect to the symmetric bilinear form $B$. It follows that $D$ is independent of the choice of the orthonomal basis $Z_{1}, \ldots, Z_{n}$ and it is invariant under the diagonal adjoint action of $K$.

The Dirac operator $D$ is a square root of Laplace operator associated to the symmetric pair $(\mathfrak{g}, \mathfrak{k})$. To explain this, we start with a Lie algebra map

$$
\alpha: \mathfrak{k} \rightarrow C(\mathfrak{p})
$$

which is defined by the adjoint map ad $: \mathfrak{k} \rightarrow \mathfrak{s o}(\mathfrak{p})$ composed with the embedding of $\mathfrak{s o}(\mathfrak{p})$ into $C(\mathfrak{p})$ using the identification $\mathfrak{s o}(\mathfrak{p}) \simeq \bigwedge^{2} \mathfrak{p}$. The explicit formula for $\alpha$ is (see $[19, \S 2.3 .3]$ )

$$
\alpha(X)=-\frac{1}{4} \sum_{j}\left[X, Z_{j}\right] Z_{j} .
$$

Using $\alpha$ we can embed the Lie algebra $\mathfrak{k}$ diagonally into $U(\mathfrak{g}) \otimes C(\mathfrak{p})$, by

$$
X \mapsto X_{\Delta}=X \otimes 1+1 \otimes \alpha(X) .
$$

This embedding extends to $U(\mathfrak{k})$. We denote the image of $\mathfrak{k}$ by $\mathfrak{k}_{\Delta}$, and then the image of $U(\mathfrak{k})$ is the enveloping algebra $U\left(\mathfrak{k}_{\Delta}\right)$ of $\mathfrak{k}_{\Delta}$.

Let $\Omega_{\mathfrak{g}}$ be the Casimir operator for $\mathfrak{g}$, given by $\Omega_{\mathfrak{g}}=\sum Z_{i}^{2}-\sum W_{j}^{2}$, where $W_{j}$ is an orthonormal basis for $\mathfrak{k}_{0}$ with respect to the inner product $-B$, where $B$ is the Killing form. Let $\Omega_{\mathfrak{k}}=-\sum W_{j}^{2}$ be the Casimir operator for $\mathfrak{k}$. The image of $\Omega_{\mathfrak{k}}$ under $\Delta$ is denoted by $\Omega_{\mathfrak{k}}$.

Then

$$
D^{2}=-\Omega_{\mathfrak{g}} \otimes 1+\Omega_{\mathfrak{k}_{\Delta}}+\left(\left\|\rho_{c}\right\|^{2}-\|\rho\|^{2}\right) 1 \otimes 1,
$$

where $\rho$ and $\rho_{c}$ are half sums of positive roots and compact positive roots respectively. 
The Vogan conjecture says that every element $z \otimes 1$ of $Z(\mathfrak{g}) \otimes 1 \subset U(\mathfrak{g}) \otimes$ $C(\mathfrak{p})$ can be written as

$$
\zeta(z)+D a+b D
$$

where $\zeta(z)$ is in $Z\left(\mathfrak{k}_{\Delta}\right)$, and $a, b \in U(\mathfrak{g}) \otimes C(\mathfrak{p})$.

A main result in [18] is introducing a differential $d$ on the $K$-invariants in $U(\mathfrak{g}) \otimes C(\mathfrak{p})$ defined by a super bracket with $D$, and determination of the cohomology of this differential complex. As a consequence, Pandžić and I proved the following theorem. In the following we denote by $\mathfrak{h}$ a Cartan subalgebra of $\mathfrak{g}$ containing a Cartan subalgebra $\mathfrak{t}$ of $\mathfrak{k}$ so that $\mathfrak{t}^{*}$ is embedded into $\mathfrak{h}^{*}$, and by $W$ and $W_{K}$ the Weyl groups of $(\mathfrak{g}, \mathfrak{h})$ and $(\mathfrak{k}, \mathfrak{t})$ respectively.

Theorem $2.3([18])$. Let $\zeta: Z(\mathfrak{g}) \rightarrow Z(\mathfrak{k}) \cong Z\left(\mathfrak{k}_{\Delta}\right)$ be the algebra homomorphism that is determined by the following commutative diagram:

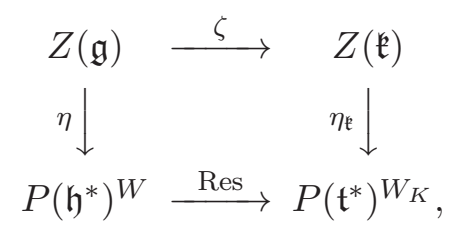

where $P$ denotes the polynomial algebra, and vertical maps $\eta$ and $\eta_{\mathfrak{k}}$ are Harish-Chandra isomorphisms. Then for each $z \in Z(\mathfrak{g})$ one has

$$
z \otimes 1-\zeta(z)=D a+a D, \text { for some } a \in U(\mathfrak{g}) \otimes C(\mathfrak{p}) .
$$

For any admissible $(\mathfrak{g}, K)$-module $X$, Vogan ([40], [18]) introduced the notion of Dirac cohomology $H_{D}(X)$ of $X$. Consider the action of the Dirac operator $D$ on $X \otimes S$, with $S$ the spinor module for the Clifford algebra $C(\mathfrak{p})$. The Dirac cohomology is defined as follows:

$$
H_{D}(X):=\operatorname{Ker} D /(\operatorname{Im} D \cap \operatorname{Ker} D) .
$$

It follows from the identity $(2.2)$ that $H_{D}(X)$ is a finite-dimensional module for the spin double cover $\widetilde{K}$ of $K$. In case $X$ is unitary, $H_{D}(X)=\operatorname{Ker} D=$ Ker $D^{2}$ since $D$ is self-adjoint with respect to a natural Hermitian inner product on $X \otimes S$. As a consequence of the above theorem, we have that $H_{D}(X)$, if nonzero, determines the infinitesimal character of $X$.

Theorem 2.4 ([18]). Let $X$ be an admissible $(\mathfrak{g}, K)$-module with standard infinitesimal character parameter $\Lambda \in \mathfrak{h}^{*}$. Suppose that $H_{D}(X)$ contains a representation of $\widetilde{K}$ with infinitesimal character $\lambda$. Then $\Lambda$ and $\lambda \in \mathfrak{t}^{*} \subseteq \mathfrak{h}^{*}$ are conjugate under $W$.

The above theorem is proved in [18] for a connected semisimple Lie group $G$. It is straightforward to extend the result to a possibly disconnected reductive Lie group in Harish-Chandra's class [8].

Vogan's conjecture implies a refinement of the celebrated Parthasarathy's Dirac inequality, which is an extremely useful tool for the classification of irreducible unitary representations of reductive Lie groups.

Theorem 2.5 (Extended Dirac Inequality [30], [18]). Let $X$ be an irreducible unitary $(\mathfrak{g}, K)$-module with infinitesimal character $\Lambda$. Fix a representation of $K$ occurring in $X$ with a highest weight $\mu \in \mathfrak{t}^{*}$, and a positive root system $\Delta^{+}(\mathfrak{g})$ for $\mathfrak{t}$ in $\mathfrak{g}$. Here $\mathfrak{t}$ is a Cartan subalgebra of $\mathfrak{k}$. Write

$$
\rho_{c}=\rho\left(\Delta^{+}(\mathfrak{k})\right), \rho_{n}=\rho\left(\Delta^{+}(\mathfrak{p})\right) .
$$


Fix an element $w \in W_{K}$ such that $w\left(\mu-\rho_{n}\right)$ is dominant for $\Delta^{+}(\mathfrak{k})$. Then

$$
\left\langle w\left(\mu-\rho_{n}\right)+\rho_{c}, w\left(\mu-\rho_{n}\right)+\rho_{c}\right\rangle \geq\langle\Lambda, \Lambda\rangle .
$$

The equality holds if and only if there exists a $w \in W$ such that

$$
\Lambda=w\left(\mu-\rho_{n}\right)+\rho_{c} .
$$

\section{DiRAC SERIES AND ELLIPTIC REPRESENTATIONS}

We say that an irreducible representation is in the Dirac series if it is a unitary representation with nonzero Dirac cohomology. The Dirac series contains several families of very important unitary representations including discrete series, unitary highest weight modules and unitary representations with nonzero $(\mathfrak{g}, K)$-cohomology. As shown in the previous section, a unitary $A_{\mathfrak{q}}(\lambda)$-module with admissible $\lambda$ is a Dirac series. Any irreducible unitary highest module is also a Dirac series. Thus, there exists Dirac series other than $A_{\mathfrak{q}}(\lambda)$-module. We now discuss the relationship between Dirac series and elliptic representations.

Let $G$ be a connected semisimple algebraic group over a local field $F$ of characteristic 0. Arthur [3] studied a subset $\Pi_{\text {temp,ell }}(G(F))$ of tempered representations of $G(F)$, namely elliptic tempered representations. The set of tempered representations $\Pi_{t e m p}(G(F))$ includes the discrete series and in general the irreducible constituents of representations induced from discrete series. These are exactly the representations which occur in the Plancherel formula for $G(F)$. In Harish-Chandra's theory $[9,10]$, the character of an infinite dimensional representation $\pi$ is defined as a distribution

$$
\Theta(\pi, f)=\operatorname{tr}\left(\int_{G(F)} f(x) \pi(x) d x\right), \quad f \in C_{c}^{\infty}(G(F)),
$$

which can be identified with a function on $G(F)$. In other words,

$$
\Theta(\pi, f)=\int_{G(F)} f(x) \Theta(\pi, x) d x, \quad f \in C_{c}^{\infty}(G(F)),
$$

where $\Theta(\pi, x)$ is a locally integrable function on $G(F)$ that is smooth on the open dense subset $G_{r e g}(F)$ of regular elements. A representation $\pi$ is called elliptic if $\Theta(\pi, x)$ does not vanish on the set of elliptic elements in $G_{r e g}(F)$.

The central objects in [3] are the normalized characters $\Phi(\pi, \gamma)$, namely the functions defined by

$$
\Phi(\pi, \gamma)=|D(\gamma)|^{\frac{1}{2}} \Theta(\pi, \gamma), \pi \in \Pi_{\text {temp }, e l l}(G(F)), \gamma \in G_{r e g}(F),
$$

where

$$
D(\gamma)=\operatorname{det}(1-\operatorname{Ad}(\gamma))_{\mathfrak{g} / \mathfrak{g}_{\gamma}},
$$

is the Weyl discriminant. We will show how this normalized character $\Phi(\pi, \gamma)$ is related to the Dirac cohomology of the Harish-Chandra module of $\pi$ for a real group $G(\mathbb{R})$.

From now on we are concerned only with the real group $G(\mathbb{R})$. Note that $G(\mathbb{R})$ has elliptic elements if and only if it is of equal rank with $K(\mathbb{R})$. We also assume this equal rank condition. Induced representations from proper parabolic subgroups are not elliptic. Consider the quotient of Grothendieck group of the category of finite length Harish-Chandra modules by the subspace generated by induced representations. Let us call this quotient group 
the elliptic Grothendieck group. Authur [3] found an orthonormal basis of this elliptic Grothendieck group in terms of elliptic tempered (possibly virtual) characters. Those characters are the super tempered distributions defined by Harish-Chandra [12].

The tempered elliptic representations for the real group $G(\mathbb{R})$ are the representations with non-zero Dirac index, which are studied in [25]. Labesse shows that the tempered elliptic representations are precisely the fundamental series. We now discuss the general elliptic representations and and their Dirac index.

Recall that if $X$ is an admissible ( $\mathfrak{g}, K)$-module with $K$-type decomposition $X=\bigoplus_{\lambda} m_{\lambda} E_{\lambda}$, then the $K$-character of $X$ is the formal series

$$
\operatorname{ch} X=\sum_{\lambda} m_{\lambda} \operatorname{ch} E_{\lambda},
$$

where $\operatorname{ch} E_{\lambda}$ is the character of the irreducible $K$-module $E_{\lambda}$. Moreover, this definition makes sense also for virtual $(\mathfrak{g}, K)$-modules $X$; in that case, the integers $m_{\lambda}$ can be negative. In the following we will often deal with representations of the spin double cover $\widetilde{K}$ of $K$, and not $K$, but we will still denote the corresponding character by ch.

Since $\mathfrak{p}$ is even-dimensional, the spin module $S$ decomposes as $S^{+} \oplus S^{-}$,

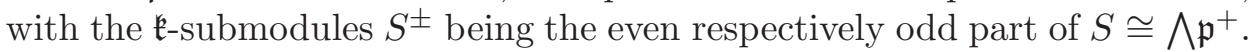
Let $X=X_{\pi}$ be the Harish-Chandra module of an irreducible admissible representation $\pi$ of $G(\mathbb{R})$. We consider the following difference of $\widetilde{K}$-modules, the spinor index of $X$ :

$$
I(X)=X \otimes S^{+}-X \otimes S^{-} .
$$

It is a virtual $\widetilde{K}$-module, an integer combination of finitely many $\widetilde{K}$-modules. The Dirac operator $D$ induces the action of the following $\widetilde{K}$-equivariant operators

$$
D^{ \pm}: X \otimes S^{ \pm} \rightarrow X \otimes S^{\mp} .
$$

Since $D^{2}$ acts by a scalar on each $\widetilde{K}$-type, most of $\widetilde{K}$-modules in $X \otimes S^{+}$are the same as in $X \otimes S^{-}$. It is straightforward to show the following identity.

Lemma 3.1. [15, Lemma 8.1] The spinor index is equal to the Euler characteristic of Dirac cohomology, i.e.,

$$
I(X)=H_{D}^{+}(X)-H_{D}^{-}(X) .
$$

The spinor index $I(X)$ is also called the Dirac index of $X$, since it is equal to the index of $D^{+}$, in the sense of index for a Fredholm operator. It is also identical to the Euler characteristic of Dirac cohomology $H_{D}(X)$. We denote by $\theta(X)$ the character of $I(X)$. In terms of characters, this reads

$$
\theta(X)=\operatorname{ch} I(X)=\operatorname{ch} X\left(\operatorname{ch} S^{+}-\operatorname{ch} S^{-}\right)=\operatorname{ch} H_{D}^{+}(X)-\operatorname{ch} H_{D}^{-}(X) .
$$

If we view $\operatorname{ch} E_{\lambda}$ as functions on $K$, then the series

$$
\operatorname{ch} X=\sum_{\lambda} m_{\lambda} \operatorname{ch} E_{\lambda}
$$

converges to a distribution on $K$ and it coincides with $\Theta(X)$ on $K \cap G_{\text {reg }}$, according to Harish-Chandra [9]. Then the absolute value $\left|\theta_{\pi}\right|$ coincides with 
the absolute value $|\Phi(\pi, \gamma)|=|D(\gamma)|^{\frac{1}{2}}|\Theta(\pi, \gamma)|$ on regular elliptic elements. We write this fact as the following lemma.

Lemma 3.2. For any regular elliptic elements $\gamma$, we have

$$
\left|\theta_{\pi}(\gamma)\right|=|\Phi(\pi, \gamma)|
$$

The following theorem follows immediately from the lemma.

Theorem 3.3. [15, Theorem 8.3] Let $\pi$ be an irreducible admissible representation of $G(\mathbb{R})$ with Harish-Chandra module $X_{\pi}$. Then $\pi$ is elliptic if and only if the Dirac index $I\left(X_{\pi}\right) \neq 0$.

We also recall a result from [15].

Theorem 3.4. [15, Theorem 10.5] Suppose $\pi$ is an irreducible unitary elliptic representation of $G(\mathbb{R})$ with a regular infinitesimal character. Then $X_{\pi} \cong A_{\mathfrak{q}}(\lambda)$.

As a consequence of the above two theorems, we have the following

Corollary 3.5. Suppose that rank of $G(\mathbb{R})$ is equal to rank of $K(\mathbb{R})$. Then any Dirac series of $G(\mathbb{R})$ with regular infinitesimal character is an $A_{\mathfrak{q}}(\lambda)$ module.

The Dirac index of a representation determines its character on compact Cartan subgroups. As shown by Harish-Chandra [12], the character of a discrete series is determined completely on the set of regular elliptic elements. It is a natural question whether the $A_{\mathfrak{q}}(\lambda)$ has the same property, namely whether the Dirac index determines the representation. It was shown in [21] that it is indeed true for most of simple Lie groups except for a few exception. In those exceptions, one needs the Dirac cohomology together with the rank of $[\mathfrak{l}, \mathfrak{l}]$ to determine the corresponding representation $A_{\mathfrak{q}}(\lambda)$.

\section{Cubic Dirac operators and associated cohomology}

We now recall the definition of Kostant's cubic Dirac operator and the basic properties of the corresponding Dirac cohomology. Let $\mathfrak{g}$ be a semisimple complex Lie algebra with Killing form $B$. Let $\mathfrak{r} \subset \mathfrak{g}$ be a reductive Lie subalgebra such that $\left.B\right|_{\mathfrak{r} \times \mathfrak{r}}$ is non-degenerate. Let $\mathfrak{g}=\mathfrak{r} \oplus \mathfrak{s}$ be the orthogonal decomposition with respect to $B$. Then the restriction $\left.B\right|_{\mathfrak{s}}$ is also non-degenerate. Denote by $C(\mathfrak{s})$ the Clifford algebra of $\mathfrak{s}$ with

$$
u u^{\prime}+u^{\prime} u=-2 B\left(u, u^{\prime}\right)
$$

for all $u, u^{\prime} \in \mathfrak{s}$. The above choice of sign is the same as in [19], but different from the definition in [23], as well as in [24]. The two different choices of signs have no essential difference since the two bilinear forms are equivalent over $\mathbb{C}$. Now fix an orthonormal basis $Z_{1}, \ldots, Z_{m}$ of $\mathfrak{s}$. Kostant [23] defines the cubic Dirac operator $D$ by

$$
D=\sum_{i=1}^{m} Z_{i} \otimes Z_{i}+1 \otimes v \in U(\mathfrak{g}) \otimes C(\mathfrak{s}) .
$$

Here $v \in C(\mathfrak{s})$ is the image of the fundamental 3-form $w \in \bigwedge^{3}\left(\mathfrak{s}^{*}\right)$,

$$
w(X, Y, Z)=\frac{1}{2} B(X,[Y, Z])
$$


under the Chevalley map $\bigwedge\left(\mathfrak{s}^{*}\right) \rightarrow C(\mathfrak{s})$ and the identification of $\mathfrak{s}^{*}$ with $\mathfrak{s}$ by the Killing form $B$. Explicitly,

$$
v=\frac{1}{2} \sum_{1 \leq i<j<k \leq m} B\left(\left[Z_{i}, Z_{j}\right], Z_{k}\right) Z_{i} Z_{j} Z_{k} .
$$

The cubic Dirac operator has a good square in analogue with the Dirac operator associated with the symmetric pair $(\mathfrak{g}, \mathfrak{k})$ in Section 2 . We have a similar Lie algebra map

$$
\alpha: \mathfrak{r} \rightarrow C(\mathfrak{s})
$$

which is defined by the adjoint map ad $: \mathfrak{r} \rightarrow \mathfrak{s o}(\mathfrak{s})$ composed with the embedding of $\mathfrak{s o}(\mathfrak{s})$ into $C(\mathfrak{s})$ using the identification $\mathfrak{s o}(\mathfrak{s}) \simeq \bigwedge^{2} \mathfrak{s}$. The explicit formula for $\alpha$ is (see $[19, \S 2.3 .3]$ )

$$
\alpha(X)=-\frac{1}{4} \sum_{j}\left[X, Z_{j}\right] Z_{j}, \quad X \in \mathfrak{r} .
$$

Using $\alpha$ we can embed the Lie algebra $\mathfrak{r}$ diagonally into $U(\mathfrak{g}) \otimes C(\mathfrak{s})$, by

$$
X \mapsto X_{\Delta}=X \otimes 1+1 \otimes \alpha(X) .
$$

This embedding extends to $U(\mathfrak{r})$. We denote the image of $\mathfrak{r}$ by $\mathfrak{r}_{\Delta}$, and then the image of $U(\mathfrak{r})$ is the enveloping algebra $U\left(\mathfrak{r}_{\Delta}\right)$ of $\mathfrak{r}_{\Delta}$. Let $\Omega_{\mathfrak{g}}$ (resp. $\Omega_{\mathfrak{r}}$ ) be the Casimir elements for $\mathfrak{g}$ (resp. $\mathfrak{r}$ ). The image of $\Omega_{\mathfrak{r}}$ under $\Delta$ is denoted by $\Omega_{\mathfrak{r}_{\Delta}}$.

Let $\mathfrak{h}_{\mathfrak{r}}$ be a Cartan subalgebra of $\mathfrak{r}$ which is contained in $\mathfrak{h}$. It follows from Kostant's calculation ([23], Theorem 2.16) that

$$
D^{2}=-\Omega_{\mathfrak{g}} \otimes 1+\Omega_{\mathfrak{r}_{\Delta}}-\left(\|\rho\|^{2}-\left\|\rho_{\mathfrak{r}}\right\|^{2}\right) 1 \otimes 1,
$$

where $\rho_{\mathfrak{r}}$ denote the half sum of positive roots for $\left(\mathfrak{r}, \mathfrak{h}_{\mathfrak{r}}\right)$. We also note the sign difference with Kostant's formula due to our choice of bilinear form for the definition of the Clifford algebra $C(\mathfrak{s})$.

We denote by $W$ the Weyl group associated to the root system $\Delta(\mathfrak{g}, \mathfrak{h})$ and $W_{\mathfrak{r}}$ the Weyl group associated to the root system $\Delta\left(\mathfrak{r}, \mathfrak{h}_{\mathfrak{r}}\right)$. The following theorem due to Kostant is an extension of Vogan's conjecture on the symmetric pair case which is proved in [18]. (See [24] Theorems 4.1 and 4.2 or [19] Theorem 4.1.4).

Theorem 4.3. There is an algebra homomorphism

$$
\zeta: Z(\mathfrak{g}) \rightarrow Z(\mathfrak{r}) \cong Z\left(\mathfrak{r}_{\Delta}\right)
$$

such that for any $z \in Z(\mathfrak{g})$ one has

$$
z \otimes 1-\zeta(z)=D a+a D \text { for some } a \in U(\mathfrak{g}) \otimes C(\mathfrak{s}) .
$$

Moreover, $\zeta$ is determined by the following commutative diagram:

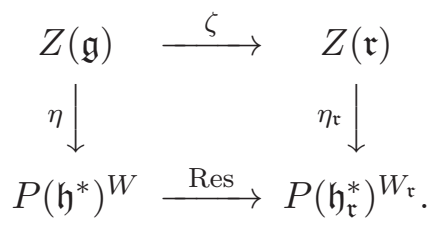

Here the vertical maps $\eta$ and $\eta_{\mathfrak{r}}$ are Harish-Chandra isomorphisms. 
Definition 4.4. Let $S$ be a spin module of $C(\mathfrak{s})$. Consider the action of $D$ on $V \otimes S$

$$
D: V \otimes S \rightarrow V \otimes S
$$

with $\mathfrak{g}$ acting on $V$ and $C(\mathfrak{s})$ on $S$. The Dirac cohomology of $V$ is defined to be the $\mathfrak{r}$-module

$$
H_{D}(V):=\operatorname{Ker} D /(\operatorname{Ker} D \cap \operatorname{Im} D) .
$$

The following theorem is a consequence of the above theorem.

Theorem 4.6 ([24],[19]). Let $V$ be a $\mathfrak{g}$-module with $Z(\mathfrak{g})$ infinitesimal character $\chi_{\Lambda}$. Suppose that an $\mathfrak{r}$-module $N$ is contained in the Dirac cohomology $H_{D}(V)$ and has $Z(\mathfrak{r})$ infinitesimal character $\chi_{\lambda}$. Then $\lambda=w \Lambda$ for some $w \in W$.

Suppose that $V_{\lambda}$ is a finite-dimensional representation with highest weight $\lambda \in \mathfrak{h}^{*}$. Kostant [24] calculated the Dirac cohomology of $V_{\lambda}$ with respect to any equal rank quadratic subalgebra $\mathfrak{r}$ of $\mathfrak{g}$. Assume that $\mathfrak{h} \subset \mathfrak{r} \subset \mathfrak{g}$ is the Cartan subalgebra for both $\mathfrak{r}$ and $\mathfrak{g}$. Define $W(\mathfrak{g}, \mathfrak{h})^{1}$ to be the subset of the Weyl group $W(\mathfrak{g}, \mathfrak{h})$ by

$$
W(\mathfrak{g}, \mathfrak{h})^{1}=\left\{w \in W(\mathfrak{g}, \mathfrak{h}) \mid w(\rho) \text { is } \Delta^{+}(\mathfrak{r}, \mathfrak{h})-\text { dominant }\right\} .
$$

This is the same as the subset of elements $w \in W(\mathfrak{g}, \mathfrak{h})$ that map the positive Weyl $\mathfrak{g}$-chamber into the positive $\mathfrak{r}$-chamber. There is a bijection $W(\mathfrak{r}, \mathfrak{h}) \times$ $W(\mathfrak{g}, \mathfrak{h})^{1} \rightarrow W(\mathfrak{g}, \mathfrak{h})$ given by $(w, \tau) \mapsto w \tau$. Kostant [24] proved the following result.

Proposition 4.7 (Kostant [24]). One has

$$
H_{D}\left(V_{\lambda}\right)=\bigoplus_{w \in W(\mathfrak{g}, \mathfrak{h})^{1}} E_{w(\lambda+\rho)-\rho_{\mathfrak{r}}}
$$

\section{ENdoscopic TRANSFER FACTOR}

Many important questions in harmonic analysis on Lie groups boil down to the study of distributions on groups that are invariant under conjugacy. The fundamental objects of invariant harmonic analysis are orbital integrals as the geometric objects and characters of representations as the spectral objects. The correspondence of these two kinds of objects reflects the core idea of harmonic analysis.

The orbital integrals are parameterized by the set of regular semisimple conjugacy classes in $G$. Recall for such a $\gamma$, the orbital integral is defined as

$$
\mathcal{O}_{\gamma}(f)=\int_{G / G_{\gamma}} f\left(x^{-1} \gamma x\right) d x, \quad f \in C_{c}^{\infty}(G),
$$

and the stable orbital integral is defined as

$$
S \mathcal{O}_{\gamma}(f)=\sum_{\gamma^{\prime} \in S(\gamma)} \mathcal{O}_{\gamma^{\prime}}(f),
$$

where $S(\gamma)$ is the stable conjugacy class. 
Let $\mathbb{1}$ denote the trivial representation of $G$ and $\theta_{\mathbb{1}}$ the character of the Dirac index of the trivial representation. That is

$$
\theta_{\mathbb{1}}=\operatorname{ch} H_{D}^{+}(\mathbb{1})-\operatorname{ch} H_{D}^{-}(\mathbb{1})=\operatorname{ch} S^{+}-\operatorname{ch} S^{-} .
$$

We note that

$$
\overline{\theta_{\mathbb{1}}}=(-1)^{q}\left(\operatorname{ch} S^{+}-\operatorname{ch} S^{-}\right)=(-1)^{q} \theta_{\mathbb{1}},
$$

where $q=\frac{1}{2} \operatorname{dim} G(\mathbb{R}) / K(\mathbb{R})$.

Recall that $\theta_{\pi}$ denotes the character of the Dirac index of $\pi$. If $\pi$ is the discrete series representation with Dirac cohomology $E_{\mu}$, then

$$
\theta_{\pi}=(-1)^{q} \chi_{\mu}
$$

Labesse showed that there exists a function $f_{\pi}$ so that for any admissible representations $\pi^{\prime}$,

$$
\operatorname{tr} \pi^{\prime}\left(f_{\pi}\right)=\int_{K} \Theta_{\pi^{\prime}}(k) \overline{\theta_{\mathbb{1}} \cdot \theta_{\pi}} d k .
$$

Denote by $\theta_{\pi^{\prime}}$ the character of its Dirac index for $\pi^{\prime}$. Then one has

$$
\operatorname{tr} \pi^{\prime}\left(f_{\pi}\right)=(-1)^{q} \int_{K} \theta_{\pi^{\prime}} \cdot \overline{\theta_{\pi}} d k .
$$

Let $\pi^{\prime}$ be a discrete series representation with Dirac cohomology $E_{\mu^{\prime}}$. It follows that

$$
\operatorname{tr} \pi^{\prime}\left(f_{\pi}\right)=\int_{K} \Theta_{\pi^{\prime}}(k) \overline{\theta_{\mathbb{1}} \cdot \theta_{\pi}} d k\left(\chi_{\mu^{\prime}}, \chi_{\mu}\right)=\operatorname{dim} \operatorname{Hom}_{K}\left(E_{\mu^{\prime}}, E_{\mu}\right) .
$$

Consequently we prove the following theorem due to Labesse.

Theorem 5.1 (Labesse [25]). The function $f_{\pi}$ is a pseudo-coefficient for the discrete series $\pi$, i.e., for any irreducible tempered representation $\pi^{\prime}$,

$$
\operatorname{tr} \pi^{\prime}\left(f_{\pi}\right)=\left\{\begin{array}{l}
1 \quad \text { if } \pi \cong \pi^{\prime} \\
0 \quad \text { otherwise. }
\end{array}\right.
$$

Remark 5.2. The orbital integrals of the pseudo-coefficient $f_{\pi}$ are easily computed for $\gamma$ regular semisimple:

$$
\mathcal{O}_{\gamma}\left(f_{\pi}\right)= \begin{cases}\Theta_{\pi}\left(\gamma^{-1}\right) & \text { if } \gamma \text { is elliptic } \\ 0 & \text { if } \gamma \text { is not elliptic. }\end{cases}
$$

In the Langlands program a cruder form of conjugacy called stable conjugacy plays an important role. The study of Langlands functoriality often leads to correspondence that is defined only up to stable conjugacy. The endoscopy theory investigates the difference between ordinary and stable conjugacy and how to understand ordinary conjugacy inside stable conjugacy. The aim is to recover orbital integrals and characters from endoscopy groups.

The endoscopy theory for real groups is established by Shelstad in a series of papers [Sh1-5]. Recasting Shelstad's work explicitly in terms of the general transfer factors defined later by Langlands and Shelstad [LS] is the first of the 'Problems for Real Groups' proposed by Arthur [5].

Recall that $G$ is a connected reductive algebraic group defined over $\mathbb{R}$. Denote by $G^{\vee}$ the complex dual group and ${ }^{L} G$ the $L$-group which is the 
semidirect product of $G^{\vee}$ and the Weil group $W_{\mathbb{R}}$. A Langlands parameter is an $L$-homomorphism

$$
\phi: W_{\mathbb{R}} \rightarrow{ }^{L} G .
$$

Two Langlands parameters are equivalent if they are conjugated by an inner automorphism of $G^{\vee}$. An equivalence class of Langlands parameters is associated to a packet of irreducible admissible representations of $G(\mathbb{R})$ [L2]. The $L$-packets of Langlands parameters with bounded image consist of tempered representations. Temperedness is respected by $L$-packets, but not unitarity.

The discrete series $L$-packets are in bijection with the irreducible finitedimensional representations of the same infinitesimal character. One can construct all tempered irreducible representations using unitary parabolic induction and by taking subrepresentations. Two tempered irreducible representations $\pi$ and $\pi^{\prime}$ are in the same $L$-packet if up to equivalence, $\pi$ and $\pi^{\prime}$ are subrepresentations of parabolically induced representations from discrete series $\sigma$ and $\sigma^{\prime}$ in the same $L$-packets.

A stable distribution is any element of the closure of the space spanned by all distributions of the form $\sum_{\pi \in \Pi} \Theta_{\pi}$ for $\Pi$ any tempered $L$-packet. Such distributions can be transferred to inner forms of $G$ via the matching of the stable orbital integrals, while unstable distributions cannot be.

In the setting of endoscopy embedding

$$
\xi:{ }^{L} H \rightarrow{ }^{L} G,
$$

one has a map from Langlands parameters for $H$ to that for $G$. The Langlands functoriality principle asserts that there should be a map from the Grothendieck group of virtual representations of $H(\mathbb{R})$ to that of $G(\mathbb{R})$, compatible with $L$-packets.

We follow Labesse $\$ 6.7[26]$ for the description of the endoscopic transfer. Let $T$ be an elliptic torus of $G$ and $\kappa$ an endoscopic character. Let $H$ be the endoscopic group defined by $(T, \kappa)$. Let $B_{G}$ be a Borel subgroup of $G$ containing $T$. Set

$$
\Delta_{B}(\gamma)=\Pi_{\alpha>0}\left(1-\gamma^{-\alpha}\right),
$$

where the product is over the positive roots defined by $B$. There is only one choice of a Borel subgroup $B_{H}$ in $H$, containing $T_{H}$ and compatible with the isomorphism $j: T_{H} \cong T$.

Assume $\eta:{ }^{L} H \rightarrow{ }^{L} G$ is an admissible embedding (see $\S 6.6[26]$ ). Then for any pseudo-coefficent $f$ of a discrete series of $G$, there is a linear combination $f^{H}$ of pseudo-coefficents of discrete series of $H$ such that for $\gamma=\gamma_{G}=j\left(\gamma_{H}\right)$ regular in $T(\mathbb{R})$ (see Prop. 6.7.1 [26]), one has

$$
\mathcal{S} \mathcal{O}_{\gamma_{H}}\left(f^{H}\right)=\Delta\left(\gamma_{H}, \gamma\right) \mathcal{O}_{\gamma}^{\kappa}(f)
$$

where the transfer factor

$$
\Delta\left(\gamma_{H}, \gamma\right)=(-1)^{q(G)-q(H)} \chi_{G, H}(\gamma) \Delta_{B}\left(\gamma^{-1}\right) \Delta_{B_{H}}\left(\gamma_{H}^{-1}\right)^{-1} .
$$

The transfer $f \mapsto f^{H}$ of the pseudo-coefficents of discrete series can be extended to all of functions in $C_{c}^{\infty}(G(\mathbb{R}))$ with extension of the correspondence $\gamma \mapsto \gamma_{H}$ (see Theorem 6.7.2 [26]) so that the above identity (5.3) holds for all $f$. 
The geometric transfer $f \mapsto f^{H}$ is dual of a transfer for representations. Given any admissible irreducible representation $\sigma$ of $H(\mathbb{R})$, it corresponds to an element $\sigma_{G}$ in the Grothendieck group of virtual representations of $G(\mathbb{R})$ as follows. Let $\phi$ be the Langlands parameter for $\sigma$. Let $\Sigma$ be the $L$ packet of the admissible irreducible representations of $H(\mathbb{R})$ corresponding to a Langlands parameter $\phi$ and $\Pi$ the L-packet of representations of $G(\mathbb{R})$ corresponding to $\eta \circ \phi$ (that can be an empty set if this parameter is not relevant for $G$ ).

Theorem 5.4 (Theorem 4.1.1 [37], Theorem 6.7.3 [26]). There is a function

$$
\epsilon: \Pi \rightarrow \pm 1
$$

such that, if we consider $\sigma_{G}$ in the Grothendieck group defined by

$$
\sigma_{G}=\sum_{\pi \in \Pi} \epsilon(\pi) \pi
$$

then the transfer $\sigma \mapsto \sigma_{G}$ satisfies

$$
\operatorname{tr} \sigma_{G}(f)=\operatorname{tr} \sigma\left(f^{H}\right) .
$$

In the following we suppose that $G(\mathbb{R})$ has a compact maximal torus $T(\mathbb{R})$, and $\rho-\rho_{H}$ the difference of half sum of positive roots for $G$ and $H$ respectively, defines a character of $T(\mathbb{R})$. In $\S 7.2$ of [Lab2] Labesse shows that the canonical transfer factor:

$$
\Delta\left(\gamma^{-1}\right)=(-1)^{q(G)-q(H)} \frac{\sum_{w \in W(\mathfrak{g})} \epsilon(w) \gamma^{w \rho}}{\sum_{w \in W(\mathfrak{h})} \epsilon(w) \gamma^{w \rho_{H}}}
$$

is well-defined function. Then the transfer factor can be expressed more explicitly if $H$ is a subgroup of $G$. Suppose that $\mathfrak{g}=\mathfrak{h} \oplus \mathfrak{s}$ is the orthogonal decomposition with respect to a non-degenerate invariant bilinear form so that the form is non-degenerate on $\mathfrak{s}$. We write $S(\mathfrak{g} / \mathfrak{h})$ for the spin-module of the Clifford algebra $C(\mathfrak{s})$. Then

$$
\Delta\left(\gamma^{-1}\right)=\operatorname{ch} S^{+}(\mathfrak{g} / \mathfrak{h})-\operatorname{ch} S^{-}(\mathfrak{g} / \mathfrak{h}) .
$$

In other words, $\Delta\left(\gamma^{-1}\right)$ is equal to the character of the Dirac index of the trivial representation with respect to the Dirac operator $D(\mathfrak{g}, \mathfrak{h})$. If $\Theta_{\pi}$ is the character of a finite-dimensional representation $\pi$, then

$$
\Delta\left(\gamma^{-1}\right) \Theta_{\pi}
$$

is the character of the Dirac index of $\pi$. This character can be calculated easily from the Kostant formula in Theorem 4.7. We denote by $F_{\lambda}$ the irreducible finite-dimensional representation of $G(\mathbb{R})$ with highest weight $\lambda$ and by $E_{\mu}$ irreducible finite-dimensional representation of $H(\mathbb{R})$ with highest weight $\mu$. Then

$$
\Delta\left(\gamma^{-1}\right) \Theta_{F_{\lambda}}=\sum_{w \in W^{1}} \Theta_{E_{w}(\lambda+\rho)-\rho_{\mathfrak{h}}} .
$$

Here $W^{1}$ is a subset of elements in $W$ corresponding to $W_{\mathfrak{h}} \backslash W$ as before.

It is straightforward to use the transfer factor to calculate lifting of discrete series characters. This lifting is closely related to the geometric transfer of the pseudo-coefficents of discrete series. The Harish-Chandra module of a discrete series representation is isomorphic to $A_{\mathfrak{b}}(\lambda)$ for some $\theta$-stable Borel 
subalgebra and corresponding Harish-Chandra parameter is $\lambda+\rho$. It follows from [8, Theorem 7.5] that a tempered representations with nonzero Dirac cohomology is $\pi_{\lambda+\rho}=A_{\mathfrak{b}}(\lambda)$ and it has Dirac cohomology equal to an irreducble $K$-module $E_{\lambda+\rho_{n}}$. In the equal rank case, it is simply a limit of discrete series. The calculation for discrete series extends to tempered representations with nonzero Dirac cohomology.

Proposition 5.5. Let $\pi_{\lambda}$ be a discrete series of $G$ with Harish-Chandra parameter $\lambda$. Then we have

$$
\Delta\left(\gamma^{-1}\right) \Theta \pi_{\lambda}=\sum_{w \in W_{K}^{1}} \operatorname{sign}(w) \Theta_{\tau_{w \lambda}} .
$$

The above formula extends to limits of discrete series.

Proof. In view of Remark 5.2, the right hand side of (5.3) is the Dirac index of a combination of discrete series of $G(\mathbb{R})$ and the left hand side is a linear combination of discrete series of $H(\mathbb{R})$. It follows from the Harish-Chandra formula for the character of discrete series and supertempered distributions [12] that the Dirac index of a discrete series $\pi_{\lambda}$ with Harish-Chandra parameter $\lambda$ is

$$
\Delta\left(\gamma^{-1}\right) \Theta \pi_{\lambda}=\sum_{w \in W_{K}^{1}} \operatorname{sign}(w) \Theta_{\tau_{w \lambda}} .
$$

Here $\tau_{w \lambda}$ denotes the discrete series for $H(\mathbb{R})$ with Harish-Chandra parameter $w \lambda$, and $W_{K}^{1}$ is a subset of elements in $W_{K}$ corresponding to $W_{H \cap K} \backslash W_{K}$. This calculation is compatible with Labesse's calculation of the transfer of the pseudo-coefficients of discrete series in $\S 7.2[26]$.

It remians to show that this formula extends to tempered elliptic representations nonzero Dirac cohomology. By Theorem 7.5 of [8] that a tempered elliptic representations nonzero Dirac cohomology is a limit of discrete sereis $\pi_{\lambda+\rho}=A_{\mathfrak{b}}(\lambda)$ and it has Dirac cohomology equal to an irreducble $K$-module $E_{\lambda+\rho_{n}}$. As the parmeter for a limit of discrete series, $\lambda+\rho$ is regular with respect to compact roots. Thus, the same calculation for discrete series applies here.

As a final remark, we note that we may use the Arthur packets to deal with non-tempered case. The Arthur packets are parameterized by mappings

$$
\psi: W_{\mathbb{R}} \times S L(2, \mathbb{C}) \rightarrow{ }^{L} G
$$

for which the projection onto the dual group $G^{\vee}$ of $\psi\left(W_{\mathbb{R}}\right)$ is relatively compact. Adams and Johnson [2] have constructed some $A$-packets consisting of unitary $A_{\mathfrak{q}}(\lambda)$-modules. As most of unitary $A_{\mathfrak{q}}(\lambda)$-modules can be classified by their Dirac cohomology [21], the determination of Dirac cohomology of $A_{\mathfrak{q}}(\lambda)$-modules may have some bearing on answering Arthur's questions $($ See $[4, \S 9])$ on Arthur packet $\Pi_{\psi}$.

\section{REFERENCES}

[1] J. Adams, Lifting of characters on orthogonal and metaplectic groups, Duke Math. J. 92 (1998), 129-178.

[2] J. Adams and J. Johnson, Endoscopic groups and packets of non-tempered representations, Compos. Math. 64 (1987), 271-309.

[3] J. Arthur, On elliptic tempered characters, Acta Math. 171 (1993), 73-138. 
[4] J. Arthur, On the Fourier transforms of weighted orbital integrals, J. Reine Angew. Math. 452 (1994), 163-217.

[5] J. Arthur, Problems for real groups, in book Representation Theory of Real Reductive Lie Groups, Contemporary Mathematics 472 (2008), 39-62.

[6] M. Atiyah, W. Schmid, A geometric construction of the discrete series for semisimple Lie groups Invent. Math. 42 (1977), 1-62.

[7] M. Atiyah, W. Schmid, Erratum: A geometric construction of the discrete series for semisimple Lie groups, Invent. Math. 54 (1979), 189-192.

[8] C.-P. Dong, J.-S. Huang, Dirac cohomology of cohomologically induced modules, Amer. J. Math. 137 (2015), 37-60.

[9] Harish-Chandra, The characters of semisimple Lie groups, Trans. Amer. Math. Soc. 83 (1956), 98-163.

[10] Harish-Chandra, Harmonic analysis on real reductive groups I. The theory of the constant term, J. Funct. Anal. 19 (1975), 104-204.

[11] Harish-Chandra, Discrete series for semisimple Lie groups, I and II, Acta Math. 113 (1965), 242-318 and 116 (1966), 1-111.

[12] Harish-Chandra, Supertempered Distributions on Real Reductive Groups, Studies in Applied Mathematics, Advances in Mathematics Supplementary Studies, 8 (1983), 139-153. (It is contained in Harish-Chandra's collected Collected Papers, Volumed IV, 447-461.)

[13] T. Howard, Lifting of characters on p-adic orthogonal and metaplectic groups, Compos. Math. 146 (2010), 795-810.

[14] R. Howe, Remarks on classical invariant theory, Trans. Amer. Math. Soc. 313 (1989), no. 2, 539-570.

[15] J.-S. Huang, Dirac cohomology, elliptic representations and endoscopy, Representation Theory of Reductive Groups, in Honor of 60th Birthday of David Vogan, Birkhauser, Progress in Mathematics, 321 (2015), 241-276.

[16] J.-S. Huang, Symplectic Dirac cohomology and lifting of characters to metaplectic groups, arXiv:2006.00157.

[17] J.-S. Huang, Y.-F. Kang and P. Pandžić, Dirac cohomology of some Harish-Chandra modules, Transform. Groups 14 (2009), no. 1, 163-173.

[18] J.-S Huang, P. Pandžić, Dirac cohomology, unitary representations and a proof of a conjecture of Vogan, J. Amer. Math. Soc. 15 (2002), 185-202.

[19] J.-S Huang, P. Pandžić, Dirac operator in Representation Theory, Math. Theory Appl., Birkhäuser, 2006.

[20] J.-S Huang, P. Pandžić, Dirac cohomology for Lie superalgebras, Transform. Groups 10 (2005), 201-209.

[21] J.-S. Huang, P. Pandžić and D.-A. Vogan, On classifying unitary representations by their Dirac cohomology, Science China Mathematics 60 (2017), 1937-1962.

[22] J.-S. Huang, P. Pandžić and F.-H. Zhu, Dirac cohomology, K-characters and branching laws, Amer. J. Math. 135 (2013), 1253-1269.

[23] B. Kostant, A cubic Dirac operator and the emergence of Euler number multiplets of representations for equal rank subgroups, Duke Math. J. 100 (1999), 447-501.

[24] B. Kostant, Dirac cohomology for the cubic Dirac operator, Studies in Memory of Issai Schur, Progress in Math. Vol. 210 (2003), 69-93.

[25] J.-P. Labesse, Pseudo-coefficients très cuspidaux et K-théorie, Math. Ann 291 (1991), 607-616.

[26] J.-P. Labesse, Introduction to endoscopy, in Representation Theory of Real Reductive Lie Groups, Contemporary Mathematics 472 (2008), 197-213.

[27] R. P. Langlands, On the classification of irreducible representations of real algebraic groups, Math. Surveys and Monographs 31 (1989), AMS, 101-170.

[28] LS R. P. Langlands, D. Shelstad, On the definition of transfer factors, Math. Ann. 278 (1987), 219-271.

[29] W.-W. Li, Spectral transfer for metaplectic groups. I. Local character relations, J. Inst. Math. Jussieu 18 (2019), 25-123.

[30] R. Parthasarathy, Dirac operator and the discrete series, Ann. of Math. 96 (1972), 1-30. 
[31] D. Renard, Endoscopy for $M p(2 n, \mathbb{R})$, Amer. J. Math. 121 (1999), 1215-1243.

[32] S. A. Salamanca-Riba, On the unitary dual of real reductive Lie groups and the $A_{\mathfrak{q}}(\lambda)$ modules: the strongly regular case, Duke Math. J. 96 (1998), 521-546.

[33] D. Shelstad, Notes on L-indistinguishability (based on a lecture by R.P. Langlands), in Automorphic Forms, Representations and L-functions, Proc. Sympos. Pure Math. 33, Part 2, Amer. Math. Soc., 1979, 193-204.

[34] D. Shelstad, Characters and inner forms of a quasi-split group over $R$, Compositio Math. 39 (1979), 11-45.

[35] D. Shelstad, Orbital integrals and a family of groups attached to a real reductive group, Ann. Sci. Ecole Norm. Sup. 12 (1979), 1-31.

[36] D. Shelstad, Embeddings of L-groups, Canad. J. Math. 33 (1981), 513-558.

[37] D. Shelstad, L-indistinguishability for real groups, Math. Ann. 259 (1982), 385-430.

[38] D. A. Vogan, Jr., Irreducible characters of semisimple Lie groups II. The KazhdanLusztig conjectures, Duke Math. J. 46 (1979), 805-859.

[39] D. A. Vogan Jr., Representations of Real Reductive Lie Groups, Progress in Math. Vol. 15, Birkhäuser, 1981.

[40] D. A. Vogan Jr., Dirac operators and unitary representations, 3 talks at MIT Lie groups seminar, Fall 1997.

[41] D. A. Vogan, Jr., G. J. Zuckerman, Unitary representations with non-zero cohomology, Compos. Math. 53 (1984), 51-90.

[42] J. A. Wolf, Partially harmonic spinors and representations of reductive Lie groups, J. Funct. Anal. 15 (1974), 117-154.

Department of Mathematics, Hong Kong University of Science and Technology, Clear Water Bay, Kowloon, Hong Kong SAR, China

Email address: mahuang@ust.hk 